\title{
An uncommon cause of chest pain
}

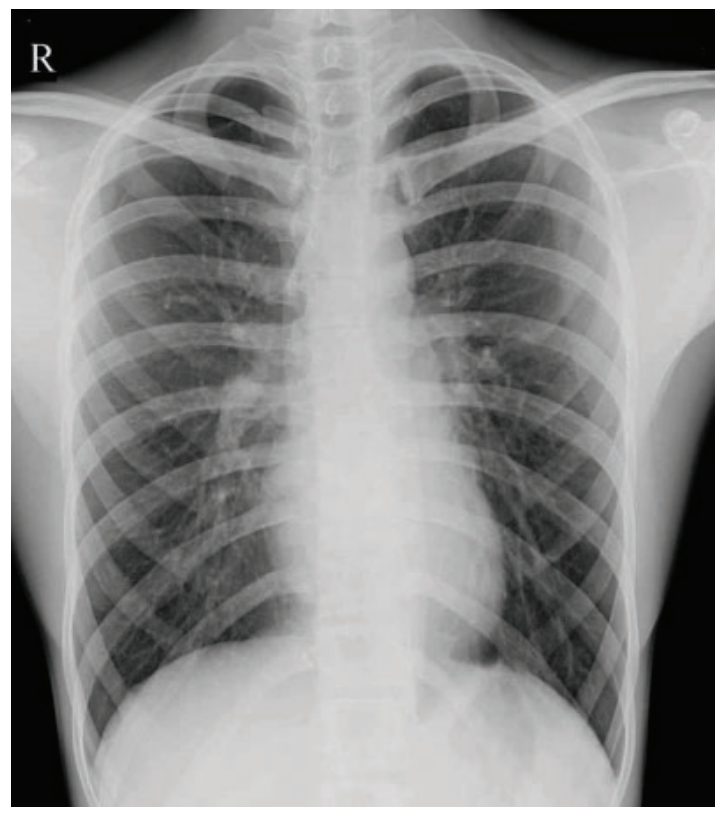

Figure 1. The chest radiograph taken at another facility was ruled to be normal.

20-Year-old man was referred to the $A$ chest pain unit with acute-onset rightsided chest pain. A chest radiograph taken at another facility (Figure 1) had been reported as normal, and electrocardiography had revealed "T inversion in lead III." The patient was referred because of this finding.

The patient had no history of significant medical illness. He was lean and thin-built. Clinical examination in the emergency department revealed no significant findings, with normal oxygen saturation on room air. Echocardiography showed no regional wallmotion abnormality, and acute coronary syndrome and aortic dissection were ruled out.

However, re-review of the chest radiograph from the previous facility noted a mild displacement of the pleural line, with absence of lung markings in the right upper lung zone, doi:10.3949/ccjm.88a.20160

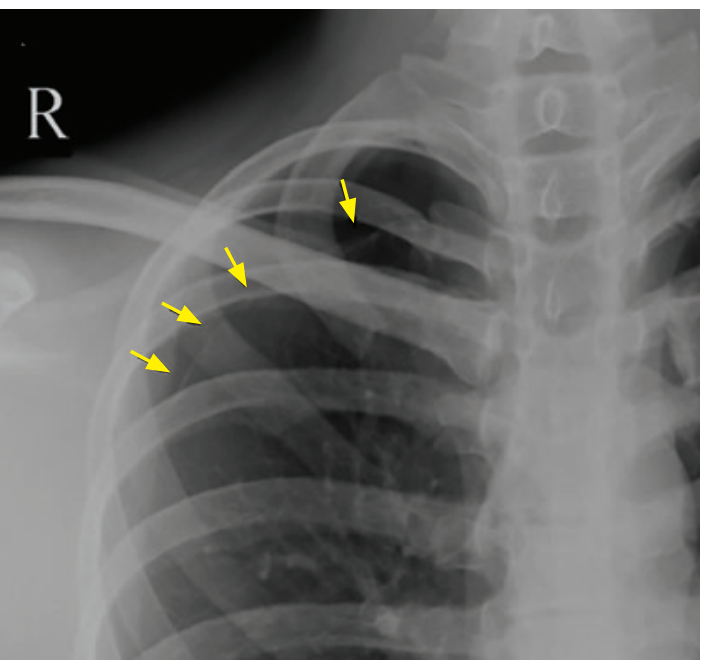

Figure 2. On re-review of the chest radiograph from another facility, magnification showed a thin visceral pleural marking (arrows) displaced from the chest wall at the right apex, indicating a small right apical pneumothorax.

suggestive of a small apical pneumothorax (Figure 2). Based on this finding, on the absence of a precipitating cause or other significant finding, and on his chest pain atypical for angina, a diagnosis of small spontaneous primary pneumothorax was made.

The patient was treated conservatively with high-flow oxygen for several hours, his cardiorespiratory status stabilized, and he was discharged.

\section{SPONTANEOUS PNEUMOTHORAX: RISK FACTORS AND DIAGNOSIS}

A small pneumothorax can be easily missed on a chest radiograph unless there is a strong index of clinical suspicion.

Pneumothorax is considered spontaneous if there is no apparent precipitating cause. Spontaneous pneumothorax is considered 
"primary" if it occurs in individuals with no underlying lung disease. Primary spontaneous pneumothorax typically occurs in tall, thin individuals. Other risk factors are male sex and cigarette smoking. Spontaneous rupture of a subpleural bleb or bulla is usually the cause.

Patients typically present with an abrupt onset of chest pain that is usually pleuritic, with or without breathlessness. Some patients may experience shoulder-tip pain.

Posteroanterior chest radiography is the standard view in patients with suspected primary spontaneous pneumothorax and may be combined with lateral radiographs in difficult cases. Expiratory films, though traditionally believed to enhance diagnostic sensitivity, may not be routinely necessary. ${ }^{1,2}$ The radiographic hallmark is displacement of the pleural line and the absence of lung markings between the edge of the pleura and chest wall. If these features are difficult to see on the posteroanterior radiograph, a lateral decubitus view with the affected side up would clearly show the lung "falling away" from the chest wall, demonstrating the pneumothorax.

Computed tomography is more sensitive in the detection of pneumothorax but is not usually required. Bedside lung ultrasonography has emerged as a new sensitive tool to diagnose pneumothorax in the emergency department, especially in trauma and critically ill patients.

\section{REFERENCES}

1. Tschopp JM, Bintcliffe $\mathbf{O}$, Astoul $\mathbf{P}$, et al. ERS task force statement: diagnosis and treatment of primary spontaneous pneumothorax. Eur Respir J 2015; 46(2):321-335. doi:10.1183/09031936.00219214

2. Bradley M, Williams C, Walshaw MJ. The value of routine expiratory chest films in the diagnosis of pneumothorax. Arch Emerg Med 1991; 8(2):115-116. doi:10.1136/emj.8.2.115

3. Baumann $\mathbf{M H}$, Strange $\mathbf{C}$, Heffner JE, et al. Management of spontaneous pneumothorax: an American College of Chest Physicians Delphi consensus statement. Chest 2001; 119(2):590-602. doi:10.1378/chest.119.2.590

\section{TREATMENT}

Conservative management with observation for 6 hours is recommended if the patient is clinically stable and no enlargement of the pneumothorax is noted on repeat radiographs. An outpatient follow-up visit 2 days after discharge is desirable. ${ }^{3,4}$

High-flow oxygen may be administered to hasten absorption of intrapleural air. ${ }^{4}$ Additional inpatient care and pleural drainage may be necessary for patients with larger pneumothoraces (distance from lung apex to visceral pleura $\geq 3 \mathrm{~cm}$, or $\geq 15 \%$ of thoracic volume $)^{3-5}$ or patients who are clinically unstable due to hypotension, respiratory distress, or significant oxygen desaturation $(<92 \%)$.

Primary spontaneous pneumothorax typically occurs at rest. Therefore, there is no need to recommend avoiding exercise to prevent recurrences.

The acute nature of the presentation can mimic an acute coronary syndrome or an acute aortic syndrome and therefore requires a strong index of suspicion for the diagnosis. A small pneumothorax can be easily missed on chest radiography and should be sought in the appropriate clinical setting.

\section{DISCLOSURES}

The author reports no relevant financial relationships which, in the context of his contributions, could be perceived as a potential conflict of interest.

4. MacDuff A, Arnold A, Harvey J; BTS Pleural Disease Guideline Group. Management of spontaneous pneumothorax: British Thoracic Society pleural disease guideline 2010. Thorax 2010; 65(suppl 2):ii18-ii31. doi:10.1136/thx.2010.136986

5. Light RW, Lee YC. Pneumothorax, chylothorax, hemothorax, and fibrothorax. In: Murray \& Nadel's Textbook of Respiratory Diseases. Philadelphia, PA: Elsevier; 2016:1439-1460.e10.

Address: Salman Salahuddin, MD, DM, MRCP (UK), Department of Cardiology, Aster Malabar Institute of Medical Sciences, Ltd., Mini Bypass Road, Govindapuram, Calicut, Kerala 673016 India; drsalmans@gmail.com 